\title{
Enhanced tunability of V-shaped plasmonic structures using ionic liquid gating and graphene
}

\author{
Onur Ozdemir a, b, *, A. Melis Aygar ${ }^{\text {a, b }}$, Osman Balci ${ }^{\text {c }}$, Coskun Kocabas ${ }^{\text {c, }}$ \\ Humeyra Caglayan b, d, Ekmel Ozbay a, b, c \\ a Department of Electrical and Electronics Engineering, Bilkent University, Ankara 06800, Turkey \\ ${ }^{\mathrm{b}}$ Nanotechnology Research Center, Bilkent University, Ankara 06800, Turkey \\ c Department of Physics, Bilkent University, Ankara 06800, Turkey \\ d Department of Electrical and Electronics Engineering, Abdullah Gul University, Kayseri 38080, Turkey
}

\section{A R T I C L E I N F O}

\section{Article history:}

Received 12 May 2016

Received in revised form 4 July 2016

Accepted 24 July 2016

Available online 25 July 2016

\begin{abstract}
A B S T R A C T
Graphene is a strong candidate for active optoelectronic devices because of its electrostatically tunable optical response. Current substrate back-gating methods are unable to sustain high fields through graphene unless a high gate voltage is applied. In order to solve this problem, ionic liquid gating is used which allows substrate front side gating, thus eliminating the major loss factors such as a dielectric layer and a thick substrate layer. On the other hand, due to its two dimensional nature, graphene interacts weakly with light and this interaction limits its efficiency in optoelectronic devices. However, V-shaped plasmonic antennas can be used to enhance the incident electric field intensity and confine the electric field near graphene thus allowing further interaction with graphene. Combining V-shaped nanoantennas with the tunable response of graphene, the operation wavelength of the devices that utilize V-shaped antennas can be tuned in situ. In the present paper, we demonstrate a graphene-based device with ionic liquid gating and $\mathrm{V}$ - shaped plasmonic antennas to both enhance and more effectively tune the total optical response. We are able to tune the transmission response of the device for up to $389 \mathrm{~nm}$ by changing the gate voltage by $3.8 \mathrm{~V}$ in the mid-infrared regime.
\end{abstract}

(c) 2016 Elsevier Ltd. All rights reserved.

\section{Introduction}

Owing to its unconventional electrical [1] and optical [2] properties, graphene has long been an active area of research. These atomically thin honeycomb lattice of carbon atoms found many uses in ultrafast electronics [3], integrated optics [4], flexible devices [5] and in the quantum world [6]. In photonics and optoelectronics, its tunable broadband interaction with the electromagnetic spectrum can be utilized in devices like photodetectors [7], optical modulators [8], polarizers [9], tunable antennas [10] and even as infrared [11] and X-ray [12] sources.

In describing the optical response of graphene, the conical band diagram is the key element used for defining the dynamics of lightgraphene interaction. Two types of band transitions are possible when a photon hits the graphene surface. Depending on the Fermi

\footnotetext{
* Corresponding author. Department of Electrical and Electronics Engineering, Bilkent University, Ankara 06800, Turkey.

E-mail address: onurozdemir@bilkent.edu.tr (O. Ozdemir).
}

level $\left(E_{f}\right)$ of graphene and energy of the incident photons, light absorption is either dominated by intraband or interband transitions. Relative effects of these transitions are defined by Pauli blocking. When the incident photon energy is lower than $2 \mathrm{E}_{\mathrm{f}}$, intraband transitions become dominant due to the lack of available states for this particular energy level at that momentum in the conduction band. At photon energies higher than $2 \mathrm{E}_{\mathrm{f}}$, interband transitions dominate $[13,14]$. Both the tunability of intrabanddominated low frequency response [15] and universal interbanddominated absorption of $2.3 \%$ [16] are demonstrated, as well as highly tunable devices operating near the $2 \mathrm{E}_{\mathrm{f}}$ energies [17].

The Fermi level of graphene is directly linked to its surface charge density, $E_{f}=\hbar v_{F} \sqrt{\pi n}$, where $\hbar$ is the reduced Planck's constant, $v_{F}$ is the Fermi velocity $\left(\sim 1.1 \times 10^{8} \mathrm{~cm} \mathrm{~s}^{-1}\right)$ and $\mathrm{n}$ is the surface charge density [18]. By changing the surface conductivity of graphene through various gating methods, Fermi level can be changed which in turn affects the optical response [19]. A mathematical model for the surface conductivity was proposed by Falkovsky, and is expressed as: 
$\sigma(\omega)=\frac{\mathrm{e}^{2} \omega}{\mathrm{i} \pi \hbar}\left[\int_{-\infty}^{+\infty} \mathrm{d} \varepsilon \frac{|\varepsilon|}{\omega^{2}} \frac{\mathrm{df}_{0}(\varepsilon)}{\mathrm{d} \varepsilon}-\int_{0}^{+\infty} \mathrm{d} \varepsilon \frac{\mathrm{f}_{0}(-\varepsilon)-\mathrm{f}_{0}(\varepsilon)}{(\omega+\mathrm{i} \delta)^{2}-4 \varepsilon^{2}}\right]$

where $\sigma$ is the complex conductivity, $\omega$ is the frequency and $\mathrm{f}_{0}(\varepsilon)=$ $\{\exp [(\varepsilon-\mu) / \mathrm{T}]+1\}^{-1}$ is the Fermi function [2]. The first term in this equation corresponds to intraband electron-photon scattering process and the second term corresponds to interband electron transitions. Integrating the intraband term leads to:

$\sigma^{i n t r a}(\omega)=\frac{2 \mathrm{ie}^{2} \mathrm{~T}}{\pi \hbar\left(\omega+\mathrm{i} \tau^{-1}\right)} \ln \left[2 \cosh \left(\frac{\mu}{2 T}\right)\right]$

where $\mathrm{T}$ is the temperature, $\mu$ is the chemical potential and $\tau$ is the electron scattering rate. The explicit dependence of intraband conductivity to the chemical potential describes the highly tunable characteristics of graphene in the intraband dominated region.

The Fermi level tuning can be controlled via hetero-atom, chemical and electrostatic doping [20], as well as unconventional methods such as plasmon-induced doping [21] and optical doping [22]. In many examples of electrically tunable graphene devices, gating is usually done via substrate back gating by using a $\mathrm{SiO}_{2}$ on $\mathrm{Si}$ substrate [17,23-25]. In this geometry, gate voltage is applied between the continuous graphene layer on one side and a metal contact on the back side of the substrate. When a gate voltage is applied between those electrodes, the voltage drop occurs mainly on the $\mathrm{SiO}_{2}$ dielectric layer whose thickness varies between 285 and $300 \mathrm{~nm}$. As a result, the electric field magnitude drops significantly near graphene, and the shift in Fermi level is decreased. An alternative gating scheme can be realized with ionic liquid gating, which enables higher magnitudes of electric field to be formed near graphene with relatively low voltages when compared to backgating methods [26]. Due to the absence of factors such as a thick dielectric layer and a much thicker substrate (although in most applications conductive doped silicon is used, there is still a voltage drop on this layer), electric field formed by the application of a gate voltage can reach to the graphene layer without such major losses. The applied voltage falls across the double layer formed at the graphene-ionic liquid interface. The double layer thickness is determined by the size of the ions $(\sim 1 \mathrm{~nm})$, which is several orders of magnitude thinner than that of the oxide $(\sim 300 \mathrm{~nm})$ used in a back gate configuration [27]. This technique enables Fermi level tuning with less voltage applied through the gating electrode.

In graphene based devices that are tuned by using an ionic liquid gating scheme, similar to the case in our paper, interband transitions become effective at photon energies higher than near infrared and visible photons $[28,29]$. As the ionic liquid is applied directly on top of the graphene layer, a chemical doping is induced on graphene. When a gate voltage is applied, ions in the ionic liquid move toward and penetrate the graphene layer, which results in a shift in Fermi level away from the Dirac point. As a result, interband transitions at mid-infrared frequencies are blocked due to Pauli blocking. The response of our device in the mid-infrared is therefore mainly due to intraband transitions.

One of the fundamental challenges in using graphene in optoelectronic applications is its relatively weak interaction with the incident field due to its atomically thin nature [16]. Echtermeyer et al. showed that plasmonic structures can be used in order to increase the interaction of graphene with the incident light by demonstrating an increase in the photocurrent of a graphene based photodetector [30]. In this paper, we have decided to use V-shaped plasmonic antennas. Among many candidates of plasmonic structures, "V"-shaped plasmonic nanoantennas have also proven useful in many applications including energy localization in nanosystems [31], quantum generation of coherent surface plasmons [32], unidirectional side scattering [33] and even sub-wavelength scale devices that can create abrupt phase changes and allows complete beam shaping [34]. The resonance frequency of the antennas used in these previous works depends on the geometry of the antennas and the substrate, therefore in situ control of the optical response was not possible. In order to achieve active control of resonance in V-shaped antennas, surface conductivity of the underlying substrate may be modified, which shifts the refractive index and the permittivity of the medium. This will allow devices which utilize Vshaped plasmonic structures to be versatile in their operating wavelength range.

In this paper, for the first time, we have experimentally demonstrated that it is possible to increase and tune the optical transmission response of a graphene based device substantially by applying less gate voltage compared to the back-gating methods via ionic liquid gating and nanoplasmonic antennas in the same device. Designing and utilizing V-shaped plasmonic structures enabled us to increase the interaction between the graphene layer and the incident field in the mid-infrared wavelengths. Moreover, by using an ionic gating scheme, we are able to induce high electric fields near graphene to efficiently tune graphene's Fermi level and control its carrier concentration, therefore shifting the transmission response of nanoantennas and the response of the device. Previous attempts that utilize substrate back-gating methods to tune graphene's Fermi level use much higher gate voltages in order to tune the optical response. Yao et al. demonstrated $650 \mathrm{~nm}$ of wavelength shift by varying the gate voltage by $26.4 \mathrm{~V}$ [35]. In a recent publication they have increased the wavelength shift up to $1100 \mathrm{~nm}$ by using $28 \mathrm{~V}$ by utilizing a different antenna design [36]. Cakmakyapan et al. demonstrated $95 \mathrm{~nm}$ of wavelength resonance shift in split-ring resonators by varying the gate voltage by $170 \mathrm{~V}$ [37]. These maximum gate voltages depend mainly on the substrate thickness and type; however, they are much higher than the gate voltage range that is used in this article, which reaches a maximum of $3.8 \mathrm{~V}$. We believe, the techniques presented in this paper will have a promising effect in designing future devices that employ graphene's tunability, as well as devices utilizing V-shaped nanoantennas. This highly effective tuning scheme may be used in future applications of actively tunable graphene based optical devices.

\section{Methods}

In order to construct the device, first of all, V-shaped plasmonic antennas with varying angles $(\alpha)$ between their two arms are fabricated on an infrared-transparent $\mathrm{BaF}_{2}$ sample using electronbeam lithography. A schematic of the device is shown in Fig. 1a. Three different types of plasmonic structures are studied. Fig. 1b shows a single plasmonic antenna that repeats itself to form a nanoantenna array on graphene. For each case, $\mathrm{L}=500 \mathrm{~nm}$ and $\mathrm{w}=50 \mathrm{~nm}$. All the structures are periodic with a period of $1 \mu \mathrm{m}$ in the $\mathrm{y}$-direction. The plasmonic antennas have a $50 \mathrm{~nm}$ of gap between their closest neighbor in the $\mathrm{x}$-direction, and the periodicity in the $\mathrm{x}$-direction is defined by this distance. The only difference between three types of structures is the angle $\alpha$ between their two arms. In our case, $\alpha$ values are selected as $90^{\circ}, 120^{\circ}$ and $150^{\circ}$ in order to investigate various geometries that $\mathrm{V}$ shaped nanoantennas can take. Scanning electron microscope (SEM) images of the final structures are shown in the inset of Fig. $2 \mathrm{a}$.

Metal coating is done via an electron-beam evaporation system and the resulting thickness of the plasmonic structures is $5 / 45 \mathrm{~nm}$ of Ti/Au. CVD grown graphene is transferred on the plasmonic structures and the substrate. To form the gating window, another $\mathrm{BaF}_{2}$ sample is patterned by photolithography to form a square 
(a)
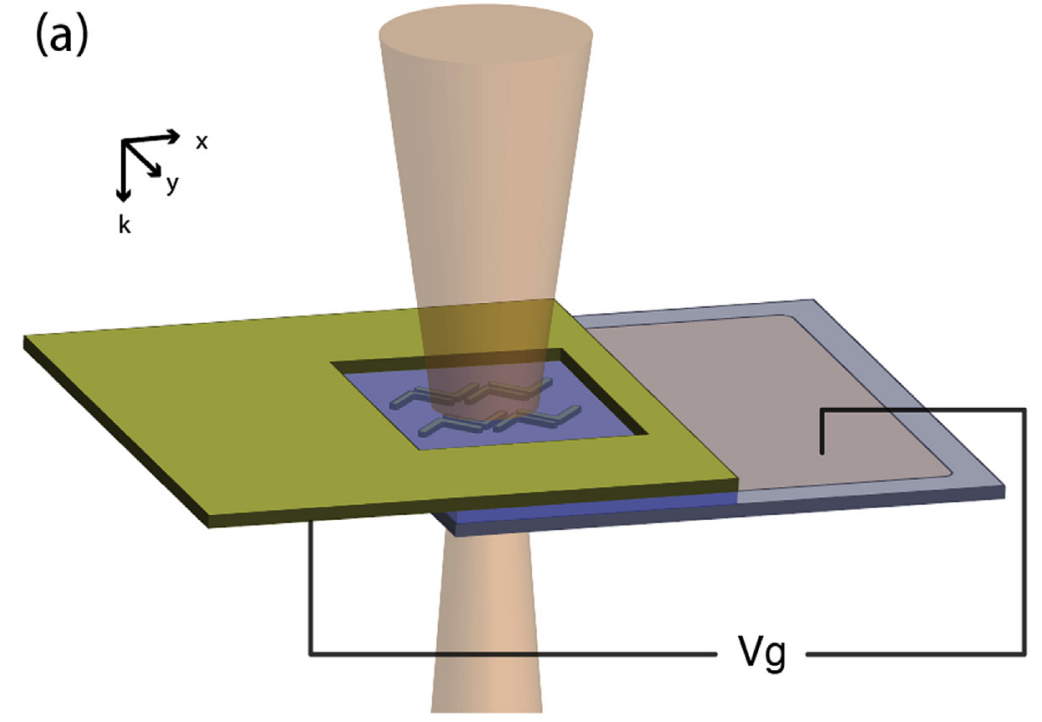

(b)

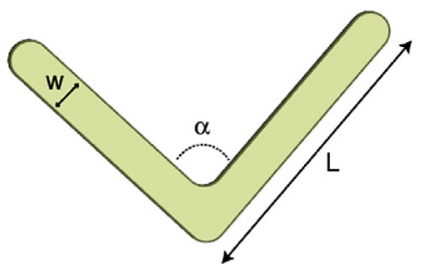

(c)

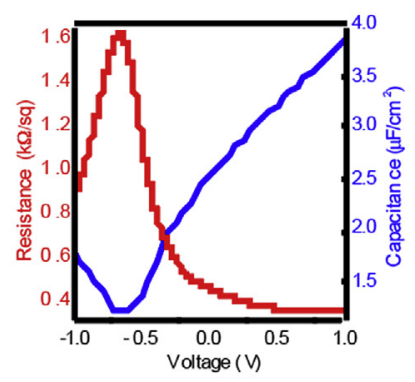

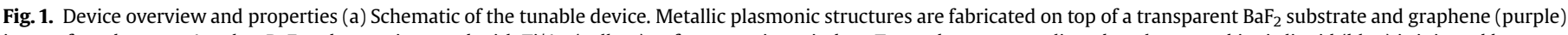

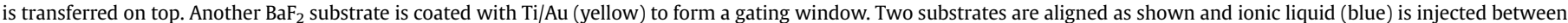

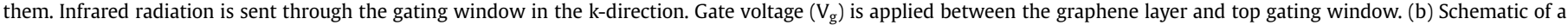

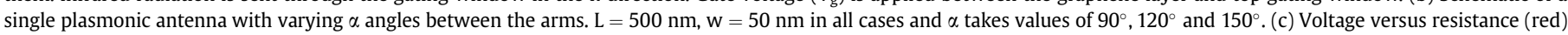

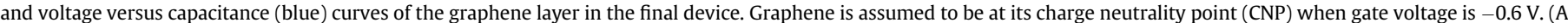
color version of this figure can be viewed online.)

opening and coated with $10 / 90 \mathrm{~nm}$ of $\mathrm{Ti} / \mathrm{Au}$ to act as a metallic top gate. Two $\mathrm{BaF}_{2}$ samples are then aligned and stabilized by using spacer bands on the sides. Gating contacts are formed by using conductive carbon bands with one being attached to a continuous graphene layer and the other to the metal coated gating window. Ionic liquid (Diethylmethyl(2-methoxyethyl) ammonium bis(trifluoromethylsulfonyl)imide, [deme][Tf2N]) is then injected in between two samples to form the final device. The thickness of the ionic liquid layer is defined by the spacer bands and is $50 \mu \mathrm{m}$.

After the device is fabricated, resistance and capacitance measurements on the graphene layer are performed in order to determine the charge neutrality point (CNP) of graphene. As graphene was transferred and the transfer process may shift graphene's Fermi level by chemical doping [38], a determination of the CNP is required for further analysis. Fig. 1c shows the measurement results of resistance and capacitance change with respect to gate voltage. Resistance reaches its maximum value and capacitance drops to its minimum value at $-0.6 \mathrm{~V}$, which is adopted to be the CNP of graphene layer for further measurements.

\section{Results and discussion}

The transmission spectra of the three different plasmonic structures (SEM images depicted on the insets) on graphene, biased by using the ionic gating method is presented in Fig. 2a. The transmission measurements are obtained by using Fourier transform infrared (FTIR) spectroscopy at different gate voltages to observe the effect of changing surface carrier concentration of graphene and its effects to the plasmonic antenna resonance wavelength. An x-polarized field is sent through the device and transmission measurements are obtained. Background measurements are taken at the gate voltage level where graphene reaches its CNP, $-0.6 \mathrm{~V}$ in an adjacent area on the device with no plasmonic structures. Gate voltage is increased in steps of $0.2 \mathrm{~V}$, up to $3.2 \mathrm{~V}$ for each case separately. When the gate voltage is increased, the Fermi level of graphene moves away from its CNP. As the surface charge density of graphene changes, the resonance wavelength of the plasmonic antennas shifts. As a result, the dips in the transmission spectra are blueshifted. Fig. 2b shows the maximum shifts of the transmission spectra for each plasmonic structure with different $\alpha$ values. Maximum spectral transmission shifts have occurred as the Fermi level of graphene moves away from the CNP; in our case, between the gate voltages of $-0.6 \mathrm{~V}$ and $3.2 \mathrm{~V}$. Transmission peaks have shifted $293.25 \mathrm{~nm}$ for $\alpha=90^{\circ}, 370.49 \mathrm{~nm}$ for $\alpha=120^{\circ}$, and up to $389.79 \mathrm{~nm}$ for $\alpha=150^{\circ}$ with a total of $3.8 \mathrm{~V}$ gate voltage tuning, in agreement with the simulations. When the gate voltage is decreased from $3.2 \mathrm{~V}$ back to $-0.6 \mathrm{~V}$, a hysteresis is observed in the dip locations of the transmission curves. This hysteresis is common to ionic-liquid based devices and is well described in both graphene-ionic liquid [39] and gold-ionic liquid [40] interfaces. One of the major mechanisms for this phenomenon is the formation of electrical double layers and slow response of the electrolyte due to low mobility of the ions in the electrolyte, which is common in supercapacitors. The other mechanism is the electrochemical doping of graphene electrodes. When a voltage is applied close to the electrochemical window of the ionic liquid, a small leakage current induces chemical doping on graphene, which shifts the CNP [15]. This mechanism may have a similar effect in our measurements. However, the tunability due to the graphene is more remarkable in our devices.

As the gate voltage increases, the shift in the spectral transmission curves diminishes. When the gate voltage is close to $3.2 \mathrm{~V}$, we see that the spectral transmission curves are almost identical. In order to further analyze the blueshift in the transmission curves and the limits of this shift, we have investigated the locations of the dips in the FTIR measurements. Fig. 3 shows the gate-voltage dependent shift of the transmission dips for 3 cases of $\alpha$. For each curve, the wavelength shifts are calculated with respect to the dip when gate voltage at the CNP. The shift values follow an almost linear trend with the increasing gate voltage up to about $2.2 \mathrm{~V}$. After that, regardless of the angle, $\alpha$, of the plasmonic structure, all curves reach a saturation in their shifts.

A proposed explanation for the diminish in the spectral transmission shifts with the increasing gate voltage focuses on the 

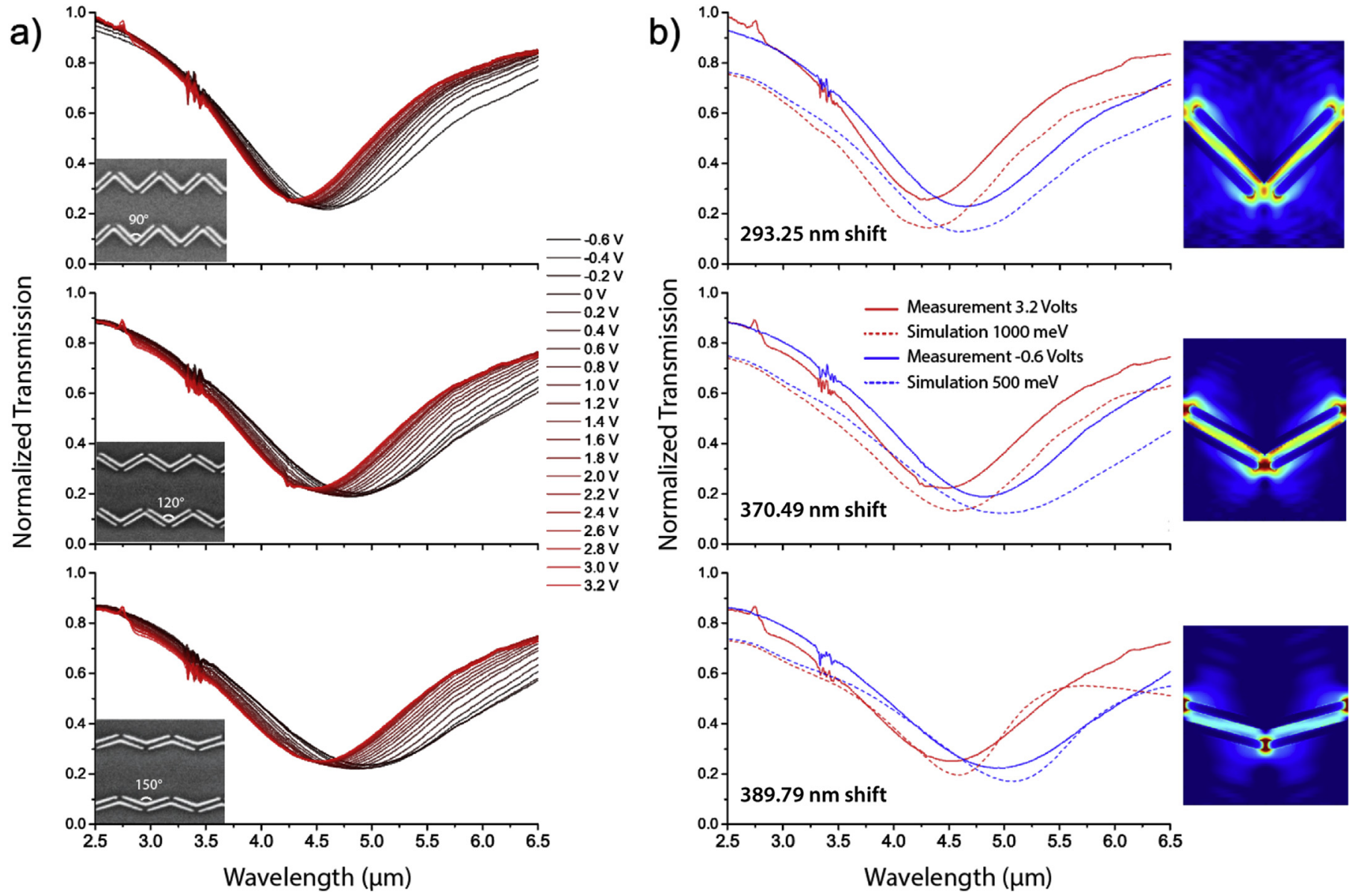

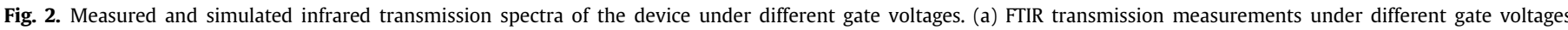

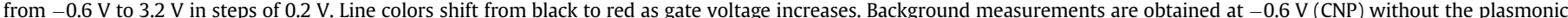

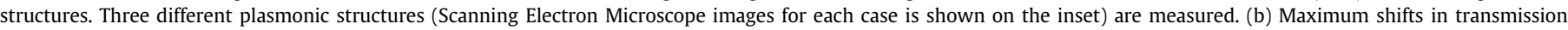

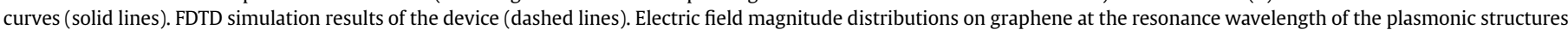

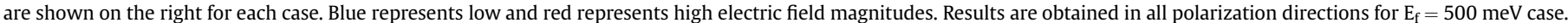
(A color version of this figure can be viewed online.)

behavior of ions in the ionic liquid. When the gate voltage increases, ions move towards the gating window and the graphene layer and accumulate in these interfaces. The accumulation of ions distorts the

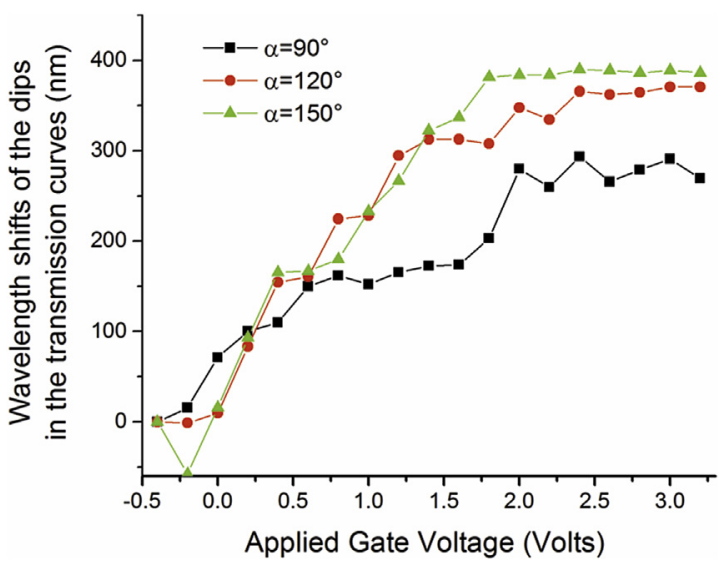

Fig. 3. Wavelength shifts of the dips in the transmission curves in Fig. 2a with respect to applied gate voltage for plasmonic structures with different values of $\alpha$. Shifts are calculated with respect to the dip in the frequency of $-0.6 \mathrm{~V}$ (assumed CNP) for each case. (A color version of this figure can be viewed online.) electric field and diminishes its magnitude near the graphene layer. This "screening" of the electric field causes the Fermi level of graphene to become less affected by the changing gate voltage. This limits the sheet carrier density change of graphene and the resonance frequency of the plasmonic modes remain unchanged.

Finite-difference time domain (FDTD) simulations are carried out using the commercially available software package Lumerical FDTD Solutions to identify the response of the device further. Graphene is introduced as a 2-dimensional dispersive material. By varying the Fermi level of the graphene layer, tunability in an optical response is achieved. The Fermi level of graphene is changed from $500 \mathrm{meV}$ to $1000 \mathrm{meV}$ in order to match the experimental curves obtained. These values are treated as fitting parameters, as I$\mathrm{V}$ measurement were not possible in our geometry, and this method has been adopted before in other publications [41] and is consistent with the results in similar geometries [28,29]. By changing the Fermi level of graphene, we are able to change the surface conductivity and effective permittivity, thereby influencing the plasmonic antenna resonance wavelength.

Further investigation of the localizations near plasmonic structures can be investigated in Figs. 2b and 4. Insets in Fig. 2b show electric field magnitude distributions on graphene layer at the resonance wavelength of the plasmonic antennas. Major localizations of the electric field are in the gaps between the long arms and 

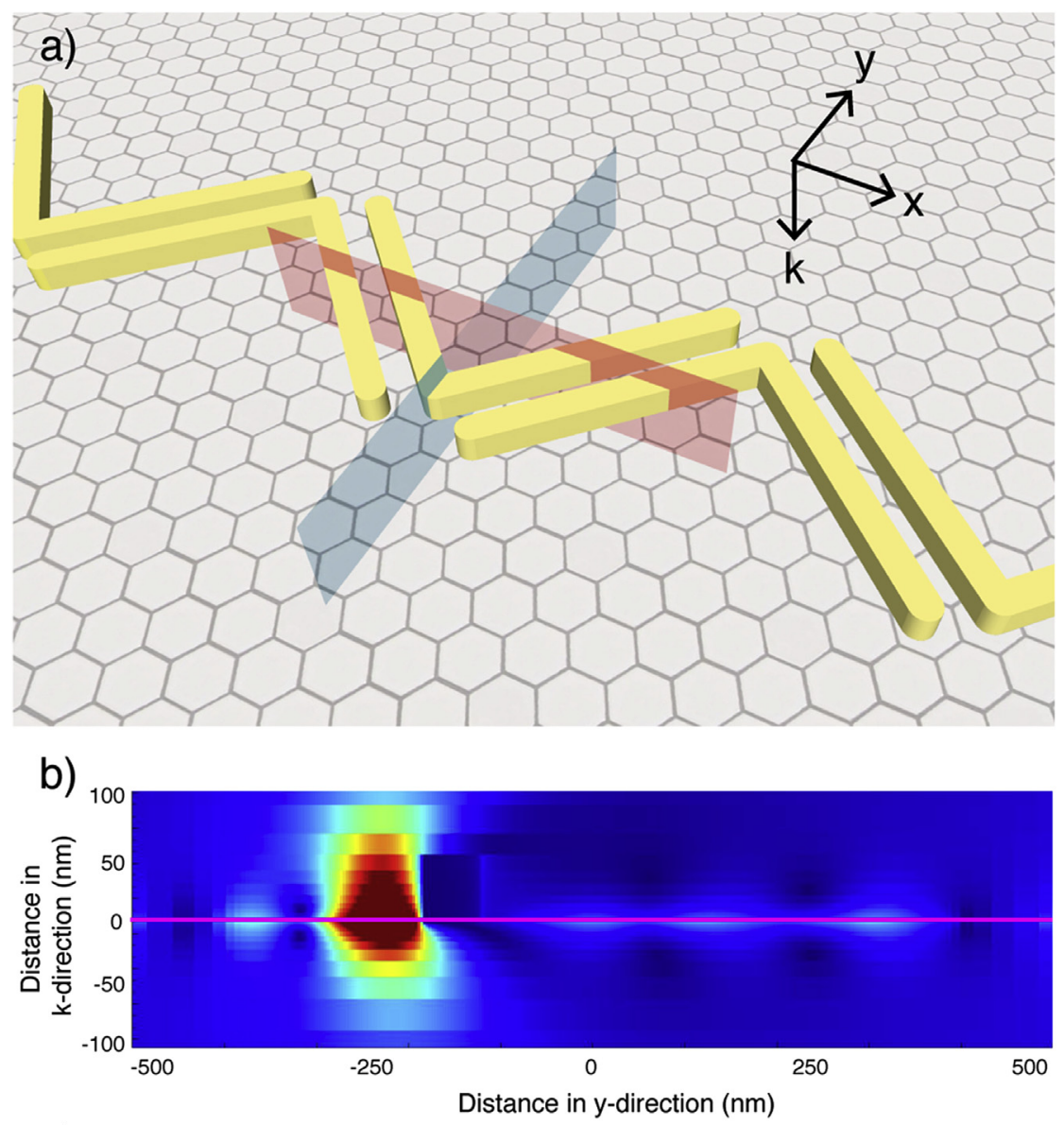

c)

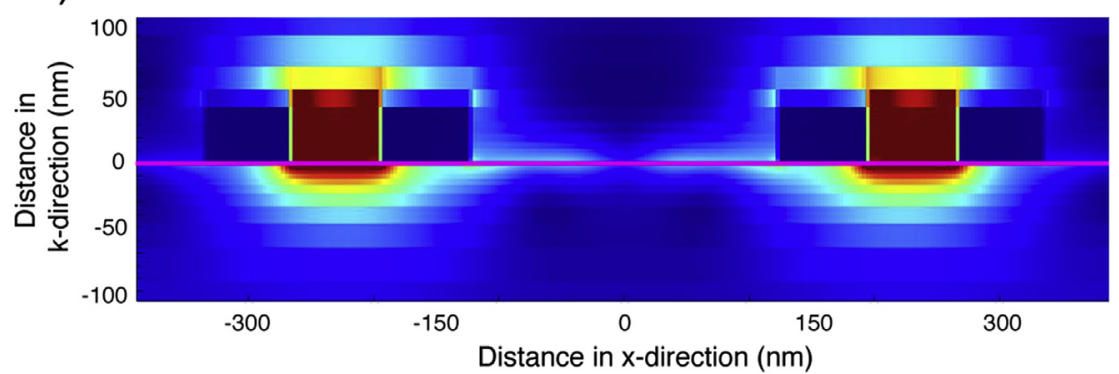

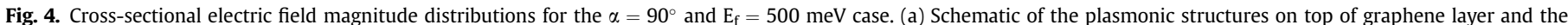

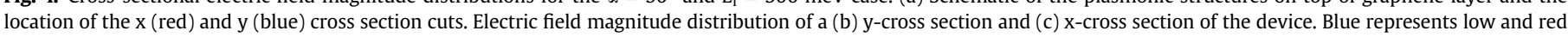

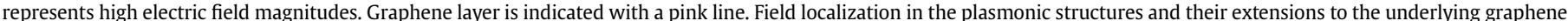
and $\mathrm{BaF}_{2}$ layers can be seen. (A color version of this figure can be viewed online.)

in the gaps between the end points of the plasmonic structures. These field localizations originate from the metallic plasmonic antennas and radiate to the nearby layers, such as graphene and $\mathrm{BaF}_{2}$ layer. The penetration of these fields into the nearby structures can be seen in Fig. 4. In each case, there is a localization of electric field on the graphene layer above the gap regions of the plasmonic antennas and that allowed further light interaction with the graphene.

\section{Conclusions}

In this article, we demonstrated a large tuning range in the transmission response of $\mathrm{V}$-shaped plasmonic antennas using graphene as the tuning medium. In addition to that, we are able to efficiently tune the spectral response of the device by applying a gate voltage in the range of $3-4 \mathrm{~V}$ through graphene via ionic liquid gating. By using this method, we are able to generate a $389 \mathrm{~nm}$ shift in the transmission spectra by applying only $3.8 \mathrm{~V}$ gate voltage. It is possible to use such devices in optoelectronic applications utilizing plasmonic antennas that require low gate voltages.

\section{Acknowledgments}

This work is supported by the projects DPT-HAMIT, NATO-SET193, TUBITAK-113E331, TUBITAK-114E374 and Research Fund of Abdullah Gül University Project Number FAB-2015-2. The authors (E.O. and H.C.) also acknowledges partial support from the Turkish Academy of Sciences. One of the authors (H.C.) also acknowledges partial support from "For Women in Science" fellowship by L'OréalUnesco Turkey. 


\section{References}

[1] A.K. Geim, K.S. Novoselov, The rise of graphene, Nat. Mater. (2007) 183-191, http://dx.doi.org/10.1038/nmat1849.

[2] L.A. Falkovsky, Optical properties of graphene, J. Phys. Conf. Ser. 129 (2008) 12004, http://dx.doi.org/10.1088/1742-6596/129/1/012004.

[3] N. Li, Z. Chen, W. Ren, F. Li, H.-M. Cheng, Flexible graphene-based lithium ion batteries with ultrafast charge and discharge rates, Proc. Natl. Acad. Sci. 109 (2012) 17360-17365, http://dx.doi.org/10.1073/pnas.1210072109.

[4] A. Pospischil, M. Humer, M.M. Furchi, D. Bachmann, R. Guider, T. Fromherz, T. Mueller, CMOS-compatible graphene photodetector covering all optical communication bands, Nat. Photonics 7 (2013) 892-896, http://dx.doi.org/ 10.1038/nphoton.2013.240.

[5] G. Eda, G. Fanchini, M. Chhowalla, Large-area ultrathin films of reduced graphene oxide as a transparent and flexible electronic material, Nat. Nanotechnol. 3 (2008) 270-274, http://dx.doi.org/10.1038/nnano.2008.83.

[6] B. Trauzettel, D.V. Bulaev, D. Loss, G. Burkard, Spin qubits in graphene quantum dots, Nat. Phys. 3 (2007) 192-196, http://dx.doi.org/10.1038/nphys544.

[7] B.Y. Zhang, T. Liu, B. Meng, X. Li, G. Liang, X. Hu, Q.J. Wang, Broadband high photoresponse from pure monolayer graphene photodetector, Nat. Commun. 4 (2013) 1811, http://dx.doi.org/10.1038/ncomms2830.

[8] W. Li, B. Chen, C. Meng, W. Fang, Y. Xiao, X. Li, Z. Hu, Y. Xu, L. Tong, H. Wang W. Liu, J. Bao, Y.R. Shen, Ultrafast all-optical graphene modulator, Nano Lett. 14 (2014) 955-959, http://dx.doi.org/10.1021/nl404356t.

[9] Y.V. Bludov, M.I. Vasilevskiy, N.M.R. Peres, Tunable graphene-based polarizer, J. Appl. Phys. 112 (2012) 84320, http://dx.doi.org/10.1063/1.4759319.

[10] N.K. Emani, T.-F. Chung, X. Ni, A.V. Kildishev, Y.P. Chen, A. Boltasseva, Electrically tunable damping of plasmonic resonances with graphene, Nano Lett. 12 (2012) 5202-5206, http://dx.doi.org/10.1021/nl302322t.

[11] V.W. Brar, M.C. Sherrott, M.S. Jang, S. Kim, L. Kim, M. Choi, L.A. Sweatlock, H.A. Atwater, Electronic modulation of infrared radiation in graphene plasmonic resonators, Nat. Commun. 6 (2015) 7032, http://dx.doi.org/10.1038/ ncomms8032.

[12] L.J. Wong, I. Kaminer, O. Ilic, J.D. Joannopoulos, M. Soljačić, Towards graphene plasmon-based free-electron infrared to X-ray sources, Nat. Photonics 10 (2015) 46-52, http://dx.doi.org/10.1038/nphoton.2015.223.

[13] K.F. Mak, L. Ju, F. Wang, T.F. Heinz, Optical spectroscopy of graphene: from the far infrared to the ultraviolet, Solid State Commun. 152 (2012) 1341-1349, http://dx.doi.org/10.1016/j.ssc.2012.04.064.

[14] V.P. Gusynin, S.G. Sharapov, J.P. Carbotte, Unusual microwave response of dirac quasiparticles in graphene, Phys. Rev. Lett. 96 (2006) 1-4, http:// dx.doi.org/10.1103/PhysRevLett.96.256802.

[15] O. Balci, E.O. Polat, N. Kakenov, C. Kocabas, Graphene-enabled electrically switchable radar-absorbing surfaces, Nat. Commun. 6 (2015) 1-9, http:// dx.doi.org/10.1038/ncomms7628.

[16] R.R. Nair, P. Blake, A.N. Grigorenko, K.S. Novoselov, T.J. Booth, T. Stauber, N.M.R. Peres, A.K. Geim, Fine structure constant defines visual transperency of graphene, Science (80-. ) 320 (2008) 2008, http://dx.doi.org/10.1126/ science.1156965.

[17] Z.Q. Li, E.A. Henriksen, Z. Jiang, Z. Hao, M.C. Martin, P. Kim, H.L. Stormer D.N. Basov, Dirac charge dynamics in graphene by infrared spectroscopy, Nat. Phys. 4 (2008) 6-9, http://dx.doi.org/10.1038/nphys989.

[18] V.E. Dorgan, M.H. Bae, E. Pop, Mobility and saturation velocity in graphene on SiO2, Appl. Phys. Lett. 97 (2010), http://dx.doi.org/10.1063/1.3483130.

[19] J. Yan, Y. Zhang, P. Kim, A. Pinczuk, Electric field effect tuning of electronphonon coupling in graphene, Phys. Rev. Lett. 98 (2007), http://dx.doi.org/ 10.1103/PhysRevLett.98.166802.

[20] B. Guo, L. Fang, B. Zhang, J.R. Gong, Graphene doping: a review, Insciences J. 1 (2011) 80-89, http://dx.doi.org/10.5640/insc.010280.

[21] Z. Fang, Y. Wang, Z. Liu, A. Schlather, P.M. Ajayan, F.H.L. Koppens, P. Nordlander, N.J. Halas, Plasmon-induced doping of graphene, ACS Nano 6 (2012) 10222-10228, http://dx.doi.org/10.1021/nn304028b.

[22] A. Tiberj, M. Paillet, P. Landois, M. Mikolasek, S. Contreras, E. Dujardin, L. Charles, M. Rubio-Roy, J.-R. Huntzinger, J.-L. Sauvajol, A.-A. Zahab, Reversible optical doping of graphene, Sci. Rep. 3 (2013) 1-23, http://dx.doi.org/ $10.1038 /$ srep02355.

[23] F. Wang, Y. Zhang, C. Tian, C. Girit, A. Zettl, M. Crommie, Y.R. Shen, Gatevariable optical transitions in graphene, Science (80-. ) 320 (2008) 206-209, http://dx.doi.org/10.1126/science.1152793.

[24] K.S. Novoselov, A.K. Geim, S.V. Morozov, D. Jiang, Y. Zhang S.V. Dubonos, I.V. Grigorieva, A.A. Firsov, Electric field effect in atomically thin carbon films, Science (80-. ) 306 (2004) 666-669, http://dx.doi.org/10.1126/ science.1102896.

[25] Y. Yao, R. Shankar, M.A. Kats, Y. Song, J. Kong, M. Loncar, F. Capasso, Electrically tunable metasurface perfect absorbers for ultrathin mid-infrared optical modulators, Nano Lett. 14 (2014) 6526-6532, http://dx.doi.org/10.1021 nl503104n.

[26] V. Thareja, J.-H. Kang, H. Yuan, K.M. Milaninia, H.Y. Hwang, Y. Cui, P.G. Kik, M.L. Brongersma, Electrically tunable coherent optical absorption in graphene with ion gel, Nano Lett. 15 (2015) 1570-1576, http://dx.doi.org/10.1021/ nl503431d.

[27] F. Chen, Q. Qing, J. Xia, J. Li, N. Tao, Electrochemical gate-controlled charge transport in graphene in ionic liquid and aqueous solution, J. Am. Chem. Soc 131 (2009) 9908-9909, http://dx.doi.org/10.1021/ja9041862.

[28] E.O. Polat, C. Kocabas, Broadband optical modulators based on graphene supercapacitors, Nano Lett. 13 (2013) 5851-5857, http://dx.doi.org/10.1021/ nl402616t.

[29] S. Balci, O. Balci, N. Kakenov, F.B. Atar, C. Kocabas, Dynamic tuning of plasmon resonance in the visible using graphene, Opt. Lett. 41 (2016) 1241, http:// dx.doi.org/10.1364/OL.41.001241.

[30] T.J. Echtermeyer, L. Britnell, P.K. Jasnos, A. Lombardo, R.V. Gorbachev, A.N. Grigorenko, A.K. Geim, A.C. Ferrari, K.S. Novoselov, Strong plasmonic enhancement of photovoltage in graphene, Nat. Commun. 2 (2011) 458, http://dx.doi.org/10.1038/ncomms1464.

[31] M.I. Stockman, S.V. Faleev, D.J. Bergman, Coherent control of femtosecond energy localization in nanosystems, Phys. Rev. Lett. 88 (2002) 67402, http:// dx.doi.org/10.1103/PhysRevLett.88.067402.

[32] D.J. Bergman, M.I. Stockman, Surface plasmon amplification by stimulated emission of radiation: quantum generation of coherent surface plasmons in nanosystems, Phys. Rev. Lett. 90 (2003) 27402, http://dx.doi.org/10.1103/ PhysRevLett.90.027402.

[33] D. Vercruysse, Y. Sonnefraud, N. Verellen, F.B. Fuchs, G. Di Martino, L. Lagae V.V. Moshchalkov, S.A. Maier, P. Van Dorpe, Unidirectional side scattering of light by a single-element nanoantenna, Nano Lett. 13 (2013) 3843-3849, http://dx.doi.org/10.1021/nl401877w.

[34] N. Yu, P. Genevet, M.a Kats, F. Aieta, J.-P. Tetienne, F. Capasso, Z. Gaburro, Light propagation with phase discontinuities reflection and refraction, Science (80) 334 (2011) 333-337, http://dx.doi.org/10.1126/science.1210713.

[35] Y. Yao, M.a Kats, P. Genevet, N. Yu, Y. Song, J. Kong, F. Capasso, Broad electrical tuning of graphene-loaded plasmonic antennas, Nano Lett. 13 (2013) 1257-1264, http://dx.doi.org/10.1021/nl3047943.

[36] Y. Yao, M.a Kats, R. Shankar, Y. Song, J. Kong, M. Loncar, F. Capasso, Wide wavelength tuning of optical antennas on graphene with nanosecond response time, Nano Lett. 14 (2014) 214-219, http://dx.doi.org/10.1021/ nl403751p.

[37] S. Cakmakyapan, H. Caglayan, E. Ozbay, Coupling enhancement of split ring resonators on graphene, Carbon N. Y. 80 (2014) 351-355, http://dx.doi.org/ 10.1016/j.carbon.2014.08.073.

[38] A. Pirkle, J. Chan, A. Venugopal, D. Hinojos, C.W. Magnuson, S. McDonnell, L. Colombo, E.M. Vogel, R.S. Ruoff, R.M. Wallace, The effect of chemical residues on the physical and electrical properties of chemical vapor deposited graphene transferred to $\mathrm{SiO}_{2}$, Appl. Phys. Lett. 99 (2011) 2-5, http:/ dx.doi.org/10.1063/1.3643444.

[39] A. Uysal, H. Zhou, G. Feng, S.S. Lee, S. Li, P. Fenter, P.T. Cummings, P.F. Fulvio, S. Dai, J.K. McDonough, Y. Gogotsi, Structural origins of potential dependent hysteresis at the electrified graphene/ionic liquid interface, J. Phys. Chem. C 118 (2014) 569-574, http://dx.doi.org/10.1021/jp4111025.

[40] T.R. Gore, T. Bond, W. Zhang, R.W.J. Scott, I.J. Burgess, Hysteresis in the measurement of double-layer capacitance at the gold-ionic liquid interface, Electrochem. Commun. 12 (2010) 1340-1343, http://dx.doi.org/10.1016 j.elecom.2010.07.015.

[41] Z. Fang, S. Thongrattanasiri, A. Schlather, Z. Liu, L. Ma, Y. Wang, P.M. Ajayan, P. Nordlander, N.J. Halas, F.J. García De Abajo, Gated tunability and hybridization of localized plasmons in nanostructured graphene, ACS Nano 7 (2013) 2388-2395, http://dx.doi.org/10.1021/nn3055835. 